\title{
A model-checking based approach: Diagnosability Verification *
}

\author{
Thiago M. Tuxi, Lilian K. Carvalho, Eduardo V. L. Nunes* \\ Antonio E. C. Da Cunha** \\ * COPPE - Programa de Engenharia Elétrica, Universidade Federal do \\ Rio de Janeiro, RJ, (e-mail: tuxi.fmce@imbel.gov.br, \\ lilian.carvalho@poli.ufrj.br,eduardo@coep.ufrj.br). \\ ** Programa de Pós-Graduação em Engenharia de Defesa (PGED), \\ Instituto Militar de Engenharia (IME), RJ (e-mail: \\ carrilho@ime.eb.br)
}

\begin{abstract}
In this work we propose a model checking approach to deal with the problem of the diagnosability verification. In this approach we consider a transition system (TS) which models the normal and the faulty behavior of the system. We propose a method to test the diagnosability property by using a linear temporal logic (LTL) formula. If a transition system does not satisfy our proposed LTL-formula, then it is not diagnosable. Our approach can be carry out in SPIN which is a tool used for formal verification of models. One advantage of SPIN is that it can handle a large state space which can be useful for diagnosability verification of complex system. Finally we present a simple example to illustrate our approach.
\end{abstract}

Keywords: Fault Diagnosis, Model-Checking, Transition Systems, Linear Temporal Logic, Discrete Event Systems.

\section{INTRODUCTION}

Failures are inherent to any real system, to ensure the safety of operation they should be diagnosed as soon as possible to avoid permanent damage to the system, low quality productions or even personal injuries.

It have been extensively applied in Continuous Variable Systems (CVS), and Discrete event Systems (DES) domains. In the context of CVS, the diagnoser infer the system conditions based on actual measurements of its variables and their expected value based on simulated models. In the later, the diagnoser infer the system condition based on observation of events.

However just a few works tries to explore this concept in a model checking paradigm Jiang and Kumar (2004, 2006); Cassez (2012). In Jiang and Kumar (2004) the system is modeled as a Transition System and the normal behavior is expressed as a linear temporal logic (LTL) formula. Failures are treated as violations of the normal behavior and are considered as permanent. In Jiang and Kumar (2006), it is extended to the case of repeated failures, it means, failures that can occur more than once. One of the main disadvantages of these approaches is due to the complexity of the construction of the Buchi Automata that recognizes the LTL formula, and despite the fact that the approach can be quite general in describing faults, it is difficult to express the normal behavior with LTL for complex systems.

Model checking is an automated technique that, given a finite-state model of a system and a formal property,

\footnotetext{
* This work is financed in part by CNPq, CAPES and FAPERJ.
}

systematically checks whether this property holds for that model (Baier and Katoen, 2008). The main idea is to examines all possible system scenarios in a systematic manner, in order to show that a given system model truly satisfies a certain property. SPIN and NUSMV which are tools used for formal verification of models and can handle state spaces up to $10^{476}$ states for specific problems. Due to this fact, it has been successfully applied in several interesting study cases, as the analysis of IEEE standards for in-house communication of domestic appliances, identification of errors in an execution module of the Deep Space 1 spacecraft controller and others.

In this work we propose a model checking approach to deal with the problem of the diagnosability verification. In this approach we consider a transition system which models the normal and the faulty behavior of the system. We propose a method to test the diagnosability property by using an LTL formula. Here, in contrast with Jiang and Kumar (2004), we consider a unique LTL formula. If a transition system does not satisfy LTL-formula, then it is not diagnosable. Our approach can be carry out in SPIN being suitable to handle complex system. Finally we present a simple example to illustrate of our approach.

\section{MODEL CHECKING PRELIMINARIES}

In this section we present the necessary concepts of model checking for our proposed approach, the reader should refer to Clarke et al. (2018); Baier and Katoen (2008) for more details. 


\subsection{Transition Systems}

A transition system $T S$ is a tuple $(S, A c t, \rightarrow, I, A P, L)$, where $S$ is a set of states, Act is a set of actions, $\rightarrow \subseteq S \times$ Act $\times S$ is a transition relation, $I \subseteq S$ is a set of initial states, $A P$ is a set of atomic propositions, and $L: \rightarrow 2^{A P}$.

For $s \in S$ and $\alpha \in$ Act the set of direct $\alpha-$ sucessors of $s$ is defined as: $\operatorname{Post}(s, \alpha)=\left\{s^{\prime} \in S \mid s \stackrel{\alpha}{\rightarrow} s^{\prime}\right\}$, $\operatorname{Post}(s)=\bigcup_{\alpha} \operatorname{Post}(s, \alpha)$.

A $T S=(S$, Act $\rightarrow, I, A P, L)$ is action - deterministic if $|I| \leq 1$ and $\mid \operatorname{Post}(s, \alpha) \leq 1$ for all $s \in S$ and $\alpha \in$ Act.

A finite execution fragment $\varrho$ of a $T S$ is an alternating sequence of states and actions ending with a state $\varrho=$ $s_{0} \alpha_{1} s_{1} \alpha_{2} \cdots \alpha_{n} s_{n}$ such that $s_{i} \stackrel{\alpha_{i+1}}{\longrightarrow} s_{i+1}$ for all $0 \leq i \leq n$.

A infinite execution fragment $\varrho$ of a $T S$ is an alternating sequence of states and actions ending with a state $\rho=$ $s_{0} \alpha_{1} s_{1} \alpha_{2} s_{3} \alpha_{3} \cdots$ such that $s_{i} \stackrel{\alpha_{i+1}}{\longrightarrow} s_{i+1}$ for all $0 \leq i$.

Let $T S_{i}=\left(S_{i}, A c t_{i}, \rightarrow_{i}, I_{i}, A P_{i}, L_{i}\right), i=1,2$ be transition systems and $H \subseteq$ Act $_{1} \cap$ Act $_{2}$ with $\tau \notin H$. The transition system $\bar{T} S_{1} \|_{H} T S_{2}$ is defined as: $T S_{1} \|_{H} T S_{2}=$ $\left(S_{1} \times S_{2}, A c t_{1} \cup A c t_{2}, \rightarrow, I_{1} \times I_{2}, A P_{1} \cup A P_{2}, L\right)$, where $L\left(\left\langle s_{1}, s_{2}\right\rangle\right)=L_{1}(s 1) \cup L_{2}\left(s_{2}\right)$, and where the transition relation $\rightarrow$ is defined as: (Baier and Katoen (2008) chapter 2).

- if $\alpha \notin H$ and $s_{1} \stackrel{\alpha}{\rightarrow}{ }_{1} s_{1}^{\prime}$ then $\left\langle s_{1}, s_{2}\right\rangle \stackrel{\alpha}{\rightarrow}\left\langle s_{1}^{\prime}, s_{2}\right\rangle$

- if $\alpha \notin H$ and $s_{2} \stackrel{\alpha}{\rightarrow}{ }_{2} s_{2}^{\prime}$ then $\left\langle s_{1}, s_{2}\right\rangle \stackrel{\alpha}{\rightarrow}\left\langle s_{1}, s_{2}^{\prime}\right\rangle$

- if $\alpha \in H$ and $s_{1} \stackrel{\alpha}{\rightarrow}_{1} s_{1}^{\prime}$ and $s_{2} \stackrel{\alpha}{\rightarrow}_{2} s_{2}^{\prime}$ then $\left\langle s_{1}, s_{2}\right\rangle \stackrel{\alpha}{\rightarrow}$ $\left\langle s_{1}^{\prime}, s_{2}^{\prime}\right\rangle$

The operator $\|_{H}$ defines the handshaking between two transition systems.

A finite path fragment $\hat{\pi}$ of $T S$ is a finite state sequence $s_{0} s_{1} \cdots s_{n}$ such that $s_{i} \in \operatorname{Post}\left(s_{i-1}\right)$ for all $0<i \leq n, 4$ where $n \geq 0$. An infinite path fragment $\pi$ is an infinite ${ }^{5}$ state sequence $s_{0} s_{1} s_{2} \cdots$ such that $s_{i} \in \operatorname{Post}\left(s_{i-1}\right)$ for all ${ }_{\mathbf{7}}^{\mathbf{6}}$ $i>0$.

The following notations is adopted for infinite path 9 fragment $\pi=s_{0} s_{1} \cdots$. For $j \geq 0$, let $\pi[j]=s_{j}$ denote the $j$ th state of $\pi$ and $\pi[\cdots j]=s_{0} s_{1} \cdots s_{j}$ denotes the 10 $j$ th prefix of $\pi$. Similarly $\pi[j \cdots]=s_{j} s_{j+1} \cdots$ denotes the ${ }^{\mathbf{1 1}}$ $j$ th suffix of $\pi$. These notions are defined analogously for 12 finite paths. Besides, for finite path $\hat{\pi}=s_{0} s_{1} \cdots s_{n}$, let 13 $\operatorname{last}(\hat{\pi})=s_{n}$ denote the last state of $\hat{\pi}$, and $\operatorname{len}(\hat{\pi})=n$ denote the length of $\hat{\pi}$.

A maximal path fragment is either a finite path fragment that ends in a terminal state, or an infinite path fragment. A path fragment is called initial if it starts in an initial state $s_{0} \in I$. A path of transition system $T S$ is an initial,maximal path fragment.

Let Path $(T S)$ denote the set of all paths in $T S$, and $\operatorname{Path}_{f i n}(T S)$ the set of all initial, finite path fragments of $T S$.

Let $T S=(S, A c t, \rightarrow, I, A P, L)$ be a transition system without terminal states. The trace of the infinite path fragment $\pi=s_{0} s_{1} \cdots$ is defined as $\operatorname{trace}(\pi)=L\left(s_{0}\right) L\left(s_{1}\right) \cdots$
The trace of the finite path fragment $\hat{\pi}=s_{0} s_{1} \cdots s_{n}$ is defined as $\operatorname{trace}(\hat{\pi})=L\left(s_{0}\right) L\left(s_{1}\right) \cdots L\left(s_{n}\right)$.

We also propose the following definitions: Let Runs $(T S)$ denote the set of all runs of TS, and Runs inf the set of all infinite runs of $T S$. The string of a run $\varrho=s_{0} \alpha_{1} s_{1} \alpha_{2} s_{2} \ldots$ is defined as string $(\varrho)=\alpha_{1} \alpha_{2} \cdots$. The projection of a string $s=\beta_{1} \beta_{2} \cdots \alpha_{1} \cdots \alpha_{2} \cdots \alpha_{n} \cdots$, with respect a set of observed actions $A c t_{o}$ is defined as $P_{o}(s)=\alpha_{1} \alpha_{2} \cdots \alpha_{n}$, where $\beta_{i} \notin A c t_{o}$ and $\alpha_{i} \in A c t_{o}$ for all $i \geq 1$. The projection erases all non observable actions of a given string.

Inspired from Cassandras and Lafortune (2008), we define the unobservable reach for a transition system $T S=$ $(S, A c t, \rightarrow, I, A P, L)$ with respect to a set of observable actions Act $_{o} \subset$ Act as:

$$
\begin{gathered}
U R(s)=\left\{p \in S: \exists \varrho=s \alpha_{1} s_{1} \alpha_{2} s_{2} \cdots p\right. \\
\left.\left[\forall i>0, \alpha_{i} \in \text { Act }_{u o} \text { and } s_{i} \stackrel{\alpha}{\rightarrow} s_{i+1} \in \rightarrow\right]\right\} \cup\{s\}
\end{gathered}
$$

In words the unobservable reach $U R(s)$, of each state $s \in S$ in a partially observed transition system is the set of all reachable states from $s$ with a succession of transitions with unobservable actions plus the state $s$ itself.

This definition can be extended to a set of states $P \subseteq S$ by $U R(P)=\bigcup_{p \in P} U R(p)$.

With these definitions we can define the observer of a Transition System $T S=\left(S, \rightarrow, A c t, s_{0}, A P, L\right)$ with respect a set of observable actions $A_{c t} \subseteq$ Act as another Transition System $T S_{o}=\left(S_{o}, \rightarrow_{o}, A_{c t}, s o_{0},\{\},\{\}\right)$ which is computed using algorithm 13, which is adapted from Cassandras and Lafortune (2008). Basically $T S_{o}$ models all runs of $T S$ that are observable with Act $_{o}$.

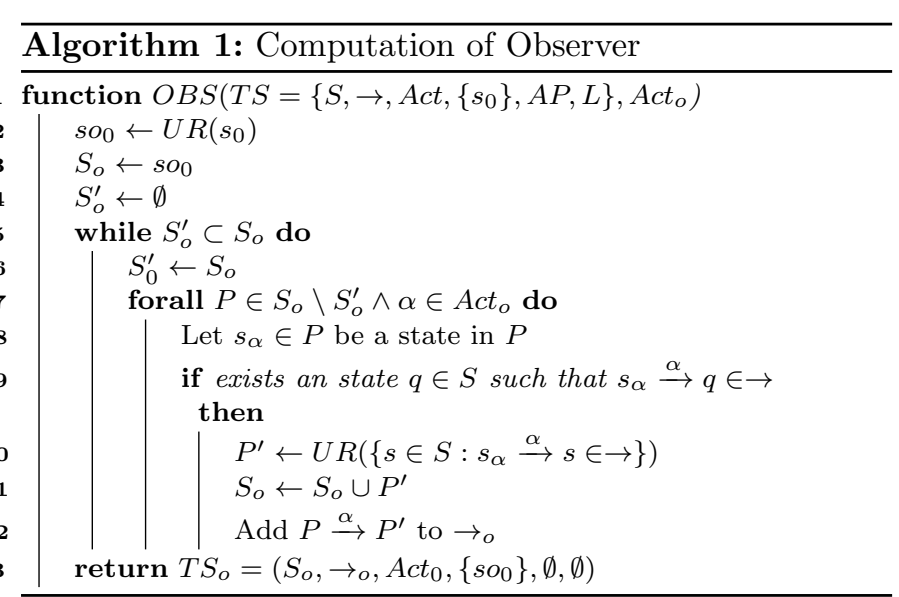

\subsection{Nondeterministic Büchi Automaton}

A nondeterministic Büchi automaton (NBA) is a tuple $\mathcal{A}=\left(Q, \Sigma, \delta, Q_{0}, F\right)$ where, $Q$ is a finite set of States, $\Sigma$ is an alphabet, $\delta: Q \times \Sigma \rightarrow 2^{Q}$ is a transition function, $Q_{0} \subseteq Q$ is a set of initial states, and $F \subseteq Q$ is a set of accept (or: final) states, called the acceptance set.

A run $\sigma=A_{0} A_{1} A_{2} \cdots \in \Sigma^{\omega}$ denotes an infinite sequence $q_{0} q_{1} q_{2} \cdots$ of states in $\mathcal{A}$ such that $q_{0} \in Q_{0}$ and $q_{1} \stackrel{A_{1}}{\longrightarrow} q_{i+1}$ for $i \geq 0$. Run $q_{0} q_{1} q_{2} \cdots$ is accepting if $q_{i} \in F$ for infinitely many index $i \in \mathbb{N}$. The accepted language of $\mathcal{A}$ is 
The size of $\mathcal{A}$ is denoted $|\mathcal{A}|=|Q|+\sum_{q \in Q \in \in} \sum_{A \in \Sigma} \mid \delta(q, A)$, is defined as the number of states and transitions in $\mathcal{A}$

The transition function $\delta$ with the induced transition relation $\rightarrow \subseteq Q \times \Sigma \times Q$ which is given by

$$
q \stackrel{A}{\rightarrow} p \text { iff } p \in \delta(q, A)
$$

Let $T S=(S, A c t, \rightarrow, I, A P, L)$ be a transition system without terminal states and $\mathcal{A}=\left(Q, 2^{A P}, \delta, Q_{0}, F\right)$ a nonblocking NBA. Then, $T S \otimes \mathcal{A}$ is the following transition system:

$$
T S \otimes \mathcal{A}=\left(S \times Q, A c t^{\prime}, \rightarrow^{\prime}, I^{\prime}, A P^{\prime}, L^{\prime}\right)
$$

where $\rightarrow^{\prime}$ is the smallest relation defined by the rule

- if $s \stackrel{\alpha}{\rightarrow} t$ and $q \stackrel{L(t)}{\longrightarrow} p$ then $\langle s, q\rangle \stackrel{\alpha}{\rightarrow}\langle t, p\rangle$

and where

- $I^{\prime}=\left\{\left\langle s_{0}, q\right\rangle \mid s_{0} \in I, \exists q_{0} \in Q_{0}: q_{0} \stackrel{L\left(s_{0}\right)}{\longrightarrow} q\right\}$

- $A P^{\prime}=Q$ and $L^{\prime}: S \times Q \rightarrow 2^{Q}$ is given by $L^{\prime}(\langle s, q\rangle)=$ $\{q\}]$

\subsection{Linear Temporal Logic}

The syntax of LTL is formed according to the following rules:

$$
\varphi::=\text { true }|a| \varphi_{1} \wedge \varphi_{2}|\neg \varphi| \bigcirc \varphi \mid \varphi_{1} U \varphi_{2}
$$

where $a \in A P$.

The until operator allows to derive the temporal modalities $\diamond$ ("eventually", sometimes in the future) and $\square$ ("always", from now on forever) as $\diamond \varphi \equiv \operatorname{true} U \varphi$ and $\square \varphi \equiv \neg \diamond \neg \varphi$. Intuitively, $\diamond \varphi$ ensures that $\varphi$ will be true eventually in the future, while $\square \varphi$ is satisfied iff it is not the case that eventually $\neg \varphi$ holds, equivalently $\varphi$ should hold from now on to forever.

Let $T S=(S, A c t, \rightarrow, I, A P, L)$ be a transitions system without terminal states, and let $\varphi$ be an LTL-formula over AP. For infinite path fragment $\pi$ of $T S$, the satisfaction relation is defined by $\pi \models \varphi$ iff $\operatorname{trace}(\pi) \models \varphi$. For state $s \in S$, the satisfaction relation $\models$ is defined by $s \models \varphi$ iff $(\forall \pi \in \operatorname{Paths}(s), \pi \models \varphi)$. TS satisfies $\varphi$, denoted by $T S \models \varphi$, if $\operatorname{Traces}(T S) \subseteq W \operatorname{ords}(\varphi)$.

\subsection{LTL Model Checking}

An LTL model-checking algorithm is a decision procedure which for a transition system $T S$ and a LTL formula $\varphi$ returns the answer "yes" if $T S \models \varphi$, and "no" (plus a counterexample) if $T S \not \models \varphi$. The counterexample consists of an appropriate finite prefix of an infinite path in $T S$ where $\varphi$ does not hold.

In the automata based model-checking approach proposed in Vardi and Wolper (1986), TS is assumed to be finite and to have no terminal states. This approach is based on the fact that each LTL formula $\varphi$ can be represented by a nondeterministic Büchi automaton (NBA). The idea is to disprove $T S \models \varphi$ by searching for a path $\pi$ in $T S$ with $\pi \models \neg \varphi$. If such a path is found, a prefix of $\pi$ is returned as an error trace. If no such path is encountered, it is concluded that $T S \models \varphi$.
The algorithm relies on the fact that $T S \models \varphi$ iff $\operatorname{Traces}(T S) \subseteq \operatorname{Words}(\varphi)$, or equivalently iff $\operatorname{Traces}(T S) \cap$ $W \operatorname{ords}(\neg \varphi)=\emptyset$.

Hence, for NBA $\mathcal{A}$ with $\mathcal{L}_{\omega}(\mathcal{A})=W \operatorname{ords}(\neg \varphi)$ we have $T S \models \varphi$ iff $\operatorname{Traces}(T S) \cap \mathcal{L}_{\omega}(\mathcal{A})=\emptyset$. Thus, to check whether $\varphi$ holds for $T S$ one first constructs an NBA for the negation of the input formula $\varphi$ and then checks for the intersection emptinesses of $\operatorname{Traces}(T S) \cap \mathcal{L}_{\omega}(\mathcal{A})=\emptyset$.

\section{MODEL CHECKING FRAMEWORK FOR DIAGNOSABILITY}

In our framework we consider that the system failures are modeled as actions and we use the atomic proposition $F$ to indicate that a failure has occurred in the system.

The Diagnosability concept proposed in Sampath et al. (1995) presents the required conditions for the construction of a diagnosis module called diagnoser, which is translated to the terminology of TS as below.

Definition 1. Let $T S=(S, A c t, \rightarrow, A P, L)$ be the transition system that model the normal and faulty behavior of the plant, with observable actions $A c t_{o} \subset$ Act, and fault actions Act $_{f} \subset A c t \backslash A c t_{o}$. Then, $T S$ is diagnosable with respect to $A c t_{f}$ and $A c t_{o}$ if and only if

$$
\begin{gathered}
\left(\forall \varrho_{1} \in \text { Runs }_{\text {inf }}(T S): \exists s_{i} \stackrel{\alpha_{i+1}}{\longrightarrow} s_{i+1}, \alpha_{i+1} \in \text { Act }_{f}\right) \Rightarrow \\
\left(\forall \varrho_{2} \in \operatorname{Runs}(T S): \nexists p_{i} \stackrel{\beta_{i+1}}{\longrightarrow} p_{i+1}, \beta_{i+1} \in \text { Act }_{f}\right) \\
\left(P_{o}\left(\operatorname{string}\left(\varrho_{1}\right)\right) \neq P_{o}\left(\operatorname{string}\left(\varrho_{2}\right)\right)\right)
\end{gathered}
$$

In words, the plant is diagnosable w.r.t to $A c t_{f}$ and $A c t_{o}$, if and only if for all arbitrary faulty long run $\varrho_{1}$ of $T S$, and all normal runs $\varrho_{2}$ of $T S,\left(P_{o}\left(\operatorname{string}\left(\varrho_{1}\right)\right) \neq P_{o}\left(\operatorname{string}\left(\varrho_{2}\right)\right)\right)$, it means that there is no uncertainty between a possible faulty run and a possible normal run of $T S$.

For verification of diagnosability of systems we split the sytem behavior in two parts, the normal behavior and the faulty behavior as below.

Definition 2. The Normal behavior of a transition system $T S=\left(S, A c t, \rightarrow,\left\{s_{0}\right\}, A P, L\right)$ with respect a set of failure actions $A_{f}$ is a transition system:

$$
T S_{N}=\left(S^{\prime}, \text { Act } \backslash A c t_{f}, \rightarrow^{\prime},\left\{s_{0}\right\}, A P=\emptyset, L=\emptyset\right)
$$
where,

- $S^{\prime}=\left\{p \in S: \exists \varrho=s_{0} \alpha_{1} s_{1} \alpha_{2} s_{2} \cdots p\left[\forall i>0, \alpha_{i} \in\right.\right.$ Act $\backslash$ Act $_{f}$ and $\left.\left.s_{i} \stackrel{\alpha}{\rightarrow} s_{i+1} \in \rightarrow\right]\right\} \cup\left\{s_{0}\right\}$;

- $\rightarrow$ is the smallest relation defined by the rule:

$$
\text { . If } s \stackrel{\alpha}{\rightarrow} p \in \rightarrow \text { and } \alpha \in A c t \backslash \text { Act }_{f} \text { then: } s \stackrel{\alpha}{\rightarrow} p \in \rightarrow^{\prime}
$$

By definition, $T S_{N}$ models all the possible non faulty behaviors of $T S$. The idea behind the construction of $T S_{N}$, is to perform a reachability analysis in $T S$, whose transitions with actions $\alpha \in$ Act $_{f}$ are not considered.

Definition 3. The Faulty behavior of a transition system $T S=\left(S, A c t, \rightarrow,\left\{s_{0}\right\}, A P, L\right)$ with respect a set of failure actions $A_{c}$ is a transition system:

$$
T S_{F}=\left(S^{\prime}, A c t, \rightarrow^{\prime},\left\{s_{0}\right\}, A P=\{F\}, L\right)
$$

where,

- $S^{\prime}=\left\{p \in S: \exists \varrho=s_{0} \alpha_{1} s_{1} \alpha_{2} s_{2} \cdots p\left[\forall i \geq 0, \alpha_{i+1} \in\right.\right.$ Act and $s_{i} \stackrel{\alpha_{i+1}}{\longrightarrow} s_{i+1} \in \rightarrow$ and $\exists i>0, \alpha_{i+1} \in$ Act $\left.\left._{f}\right]\right\}$ 
- $\rightarrow^{\prime}$ is the smallest relation defined by the rule: - If $s \stackrel{\alpha}{\rightarrow} p \in \rightarrow$ and $s, p \in S^{\prime}$ then: $s \stackrel{\alpha}{\rightarrow} p \in \rightarrow^{\prime}$

By definition, $T S_{F}$ models all the possible faulty behaviors of $T S$. The idea behind the construction of $T S_{F}$, is to perform a reachability analysis in $T S$, recording all runs of $T S$ that has at least one transition with an action $\alpha \in$ Act $_{f}$.

We also define $T S_{N_{O b s}}^{c o m p l}$, which is a transition system that models all the observable runs that goes out of the normal behavior of the system. We attribute an atomic proposition $D$ to indicate that a run has escaped from the observable normal behavior of the system, in other words, it indicates that we identified that a fault has occurred.

Algorithm 2 is used to compute $T S_{F}$ and $T S_{N o b s}^{c o m p l}$. At lines 2 and 3 we compute $T S_{N}$ from $T S$ and $A c t_{f}$ and $T S_{F}$ from $T S$ and $A c t_{f}$. At line 4 we compute $T S_{N_{O b s}}$, the observer of $T S_{N}$. At line 5 we complete $T S_{N_{O b s}}$ with a dummy state $A l$ which is a state that indicates that we detected an ocurrence of a failure. At line 6 , we atribute an atomic proposition $D$ to state $A l$. Finally, at line 7 we rename $T S_{N_{O b s}}$ to $T S_{N_{O b s}}^{c o m p l}$.

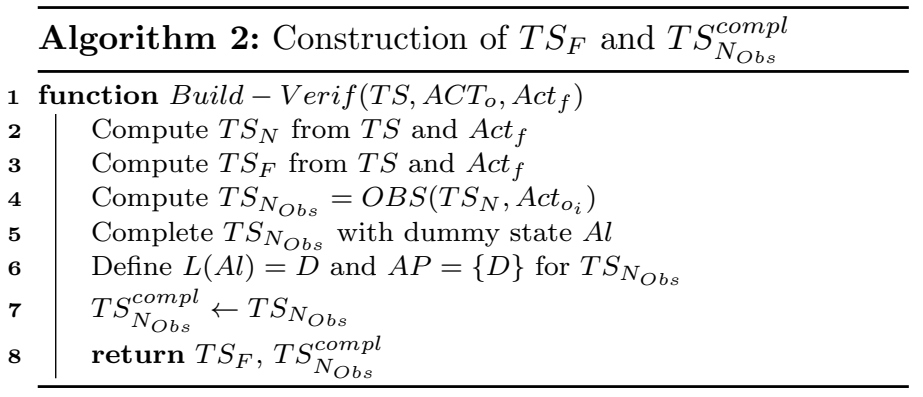

Example 1. In order to illustrate the application the method presented in this section, we present the example illustrated in figure 1.

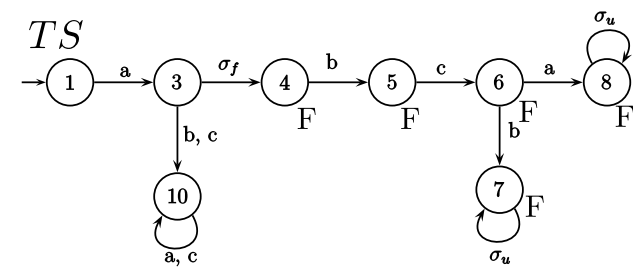

Figure 1. Example of a non diagnosable system

In this example we consider the verification of the diagnosability of $T S$ with the set of observations $A c t_{o}=$ $\{a, b\}$, to diagnose failure $\sigma_{f} . T S_{N}, T S_{N_{O b s}}$ and the proposed diagnosis module $T S_{N_{O b s}}^{c o m p l}$ are illustrated in figure 2. $T S_{F}$ is illustrated in figure 3 .

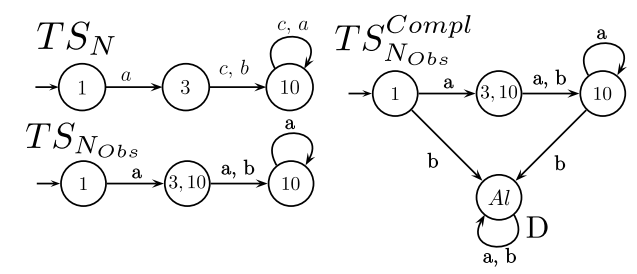

Figure 2. Construction of the diagnosis module

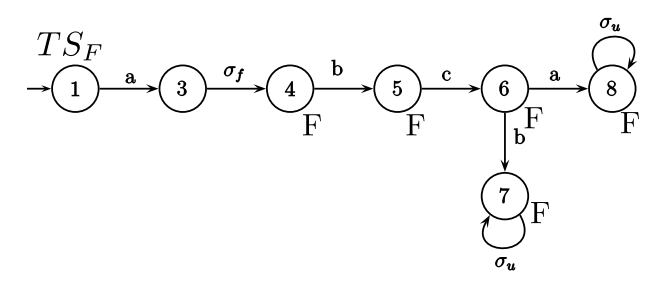

Figure 3. TS that models the faulty behavior of the system.

The interaction between $T S_{F}$ and $T S_{N_{O b s}}^{c o m p l}$ will be further discussed and used to derive our model checking approach to verify the diagnosability of $T S$ in the next section.

\section{DIAGNOSABILITY VERIFICATION VIA MODEL CHECKING}

The diagnosability of a system is the necessary and sufficient condition to design a diagnosis module that complies to the following two properties:

(1) The module should never issue false alarms;

(2) If a fault happen in the system then the module should detect it in the future.

Which can be easily expressed as LTL formula as below:

(1) $\square(\neg F \rightarrow \neg D)$

(2) $\square(F \rightarrow \diamond D)$

where, $F$ and $D$ are atomic propositions that indicate that a fault has occured and that the system has diagnosed it, respectively.

For a given TS which has the normal and faulty behavior of the system, it's always possible to construct a diagnosis module that attends the first requirement by construction, since we consider that we know a priori all the possible normal and faulty runs. Informally, the module should issues an alarm if and only if it detects runs that are not feasible in normal conditions of the system.

In our approach like in standard ones for diagnosis of DES, the $T S_{F}$ is responsible to generate actions (events), the $T S_{N_{O b s}}^{c o m p l}$ only observes the sequences generated by the $T S_{F}$ without interfering with its behavior. In order to model this kind of interaction, we use the handshaking product as presented in section 2, computing $T S_{V}=T S_{F} \|_{H}$ Diag, where $H=A c t_{o}$. The idea is that observable actions of the plant are executed synchronously in both transition systems, while unobservable actions of occur freely in $T S_{F}$. Note that in $T S_{N_{O b s}}^{c o m p l}$, observable actions are always enabled, thus, there are no blockage of $T S_{F}$ actions.

The last step is to check if $T S_{V} \models \square(F \rightarrow \diamond D)$ using model checking, if positive the plant is diagnosable, otherwise the plant is not diagnosable, note that in our approach the diagnosability is tested in a indirect way. We apply the model checking technique presented in section 2 to try disprove $T S_{V}=\square(F \rightarrow \diamond D)$, for this we compute the Buchi automaton for the negation of $\varphi=\square(F \rightarrow \diamond D)$, as shown in figure 4 .

Algorithm 3 is used to perform our model checking approach. At line 2 we compute $T S_{V}=T S_{F} \|_{A c t_{o}} T S_{N_{O b s}}^{\text {compl }}$ which models the interaction of the observed normal behavior with the faulty behavior of the plant. At line 


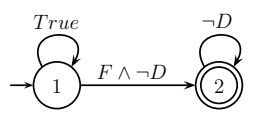

Figure 4. Buchi Automaton for $\diamond(F \wedge \square \neg D)$

3 to 8 we apply the model checking technique presented in section 2 to try disprove $T S_{V} \models \square(F \rightarrow \diamond(D)$, if the LTL formula is disproved we output a counter example and inform that the system is not diagnosable, otherwise the system is diagnosable.

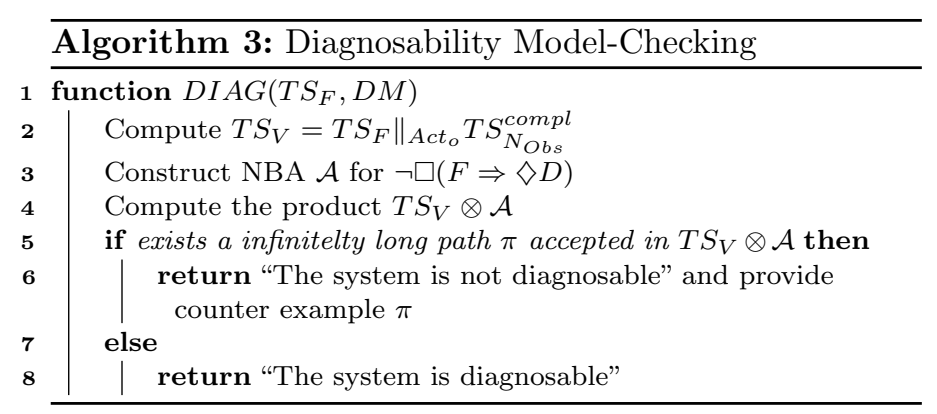

The following theorem states the correctness of our method.

Theorem 1. Let $T S_{V}$ be the transition system that model the interaction of the faulty behavior of the plant $T S$ with the proposed diagnosis module $T S_{N_{O b s}}^{c o m p l}$ and $\varphi=\square(F \rightarrow$ $\diamond D)$. Then, $T S$ is diagnosable w.r.t to $A_{c t}$ and $A_{c} t_{f}$ if and only if $T S_{V} \models \varphi$

We briefly discuss the complexity of our algorithm, the complexity to compute $T S_{N}$ and $T S_{F}$ is linear in the size of $T S$, the complexity to compute $O B S\left(T S_{N}, A c t_{o}\right)$ is $2^{|S|}$, it occurs due to the subset construction involved in the agregation of states in the unobservable reach of states of $T S_{N}$, the complexity to compute Diag is linear in the size of $O B S\left(T S_{N}, A c t_{o}\right)$. The handshaking product $T S_{V}$ is linear in the size of $T S_{F}$, note that the handshaking only aggregates an atomic proposition $D$ to each state of $T S_{F}$. The complexity of construction of NBA $\mathcal{A}$ is fixed since the number of until operators is fixed. The cost to perfom model checking then is linear in the size of $T S_{V}$. Then the worst case complexity of our proposed model checking is $2^{|S|}$, which is the complexity of computation of the observer. Note however that, in practical situations the size of the observer is quite smaller than the worst case scenario.

Example 2. In order to illustrate the application of our model checking approach to verify the diagnosability of systems, we apply algorithm 3 in Example 1 presented before.

The Handshaking $T S_{V}=T S_{F} \|_{H} D M$, with $H=$ Act $_{o}$, that models the interaction between the faulty runs of the plant $T S_{F}$ and the observed normal behavior of the plant $T S_{N_{O b s}}^{c o m p l}$ is illustrated in figure 5 .

The next step is the verification if $T S_{V} \models \square(F \rightarrow \diamond D)$, the construction of the Buchi automata $\mathcal{A}$ for $\neg \varphi$ is shown in figure 4 and the product $T S_{V} \otimes \mathcal{A}$ is shown in figure 6 . It is easy to check that the infinitely long path $\pi=$ $(1,1,1)(3,\{3,10\},\{1,2\})(3,\{3,10\},\{1,2\})(5,10,\{1,2\})$ $(6,10,\{1,2\})(8,10,\{1,2\})(8,10,\{1,2\}) \cdots$ is accepted in

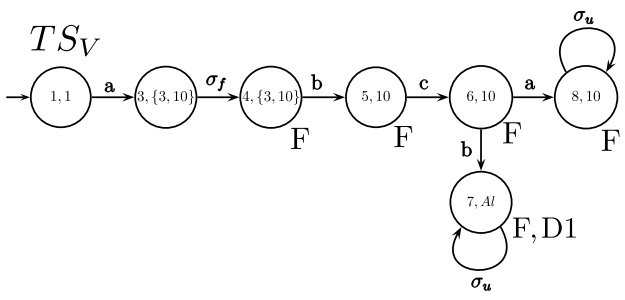

Figure 5. Handshaking interaction between the plant and the diagnosis module

$T S_{V} \otimes \mathcal{A}$, with the respecting string $a \sigma_{f} b c a \sigma_{u}^{\omega}$, thus, the system is not diagnosable w.r.t $A c t_{f}$ and $A c t_{o}$.

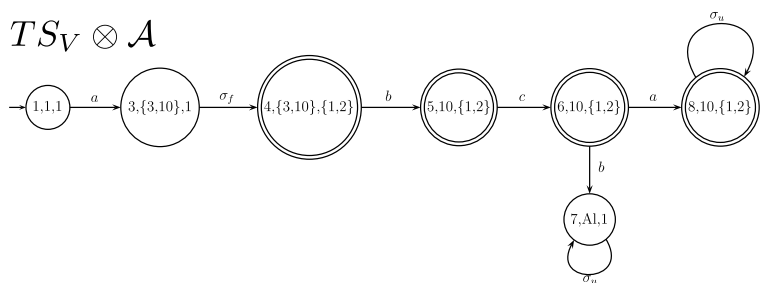

Figure 6. Product of $T S_{V}$ and Buchi automaton $\mathcal{A}$

In the next section we will illustrate a method to implement algorithm 3 in the SPIN model checking tool.

\section{SPIN IMPLEMENTATION}

In this section we present our implementation method of our algorithm in SPIN to check if $T S_{V} \models \square(F \rightarrow \diamond D)$ by means of an application example as the one presented in section 4. SPIN uses a proprietary programming language called Promela (Process meta language) that allows the creation of models of concurrent processes, for more information regarding this programming language, the reader should refer to the Promela reference manual at http://spinroot.com/spin/Man/Intro.html .

It's important to note that in SPIN the interaction between processes can be defined trough channels in a synchronous or asynchronous way. In our approach we define the communications in a synchronous way, it means that once the plant issues an action, the diagnosis module will listen to it without delay. In this context we define that $T S_{F}$ is responsible to generate and transmits actions trough a channel and $T S_{N_{O b s}}^{c o m p l}$ listen and reacts to the actions received via the same channel.

In our implementation framework, we define Actions as an mtype declaration which allows the introduction of symbolic names for constant values, atomic propositions $\mathrm{F}$ and $\mathrm{D}$ are declared as Boolean variables. TS will be declared as proctype constructs with the prefix active that indicates that this process is automatically started at run time. The synchronous communication channel between $T S_{F}$ and $T S_{N_{O b s}}^{c o m p l}$ is declared with the command chan F2D $=[0]$ of mtype . The LTL formula $\varphi=\square(F \rightarrow \diamond D)$ is expressed with the command ltl L1 []$(\mathrm{F}-><>\mathrm{D})$;

The idea of our implementation is, given $T S_{F}, T S_{N_{0 b}}^{c o m p l}$, and the LTL formula $\varphi=\square(F \rightarrow \diamond D)$, SPIN internally computes $T S V$ and verify if $T S_{V} \models \square(F \rightarrow$ $\diamond D)$, if $T S_{V} \forall \square(F \rightarrow \diamond D)$ then SPIN generates 
an counterexample for verification purposes. The Buchi automaton $\mathcal{A}$ and the product $T S_{V} \otimes \mathcal{A}$ are also computed internally on SPIN.

One of the advantages of this approach is that SPIN does not explicitly constructs the entire state space of $T S_{V}$, it construct it on the fly during verification. If a counterexample is found the verification is stopped, another advantage is that as mentioned before, SPIN can handle system with up to $10^{476}$ states. Part of the Promela code used for declaration of the system presented in example 1 is illustrated below, showing the definition of the actions, the atomic propositions, the synchronous communication channel, the LTL formula and part of $T S_{F}$. We define two processes, active proctype TSF and active proctype DM, and we define LTL formula L1 for verification of diagnosability. For illustration purposes we assume that the code is saved in Exemplo1.pml file. The SPIN files for this example can be found at https:// github.com/TuxiThiago/SPIN_FDIAGNOSIS.

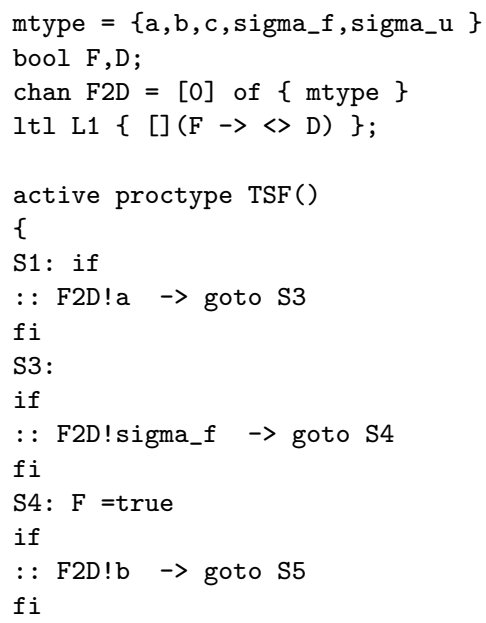

We use the following commands to compile the verifier for the system declared in Exemplo1.pml.

Console input: spin -a Exemplo1.pml

Console output: ltl L1: [] $((!(F))||(<>(D)))$

Console input: gcc -o pan pan.c

Then we run the verifier with the $/$ pan $-a$ command. For this example, the system is not diagnosable, the two first lines of the output indicates that the LTL formula L1 was violated, and that the counter example was saved at Exemplo1.pml.trail, the remaining lines of the output are omitted due to space limitations.

Console input: ./pan -a

Console output: pan:1: acceptance cycle (at depth 21)

pan: wrote Exemplo1.pml.trail

We then check the counter example with the spin - $t$ c Exemplo1.pml command. A run that violates the LTL formula will be printed, in this case the string $a \sigma_{f} b c a \sigma_{u}^{\omega}$ violates the LTL formula $\mathrm{L} 1$. This result matches the result obtained in example 1 in section 4. Part of the counterexample provided by SPIN is presented below.

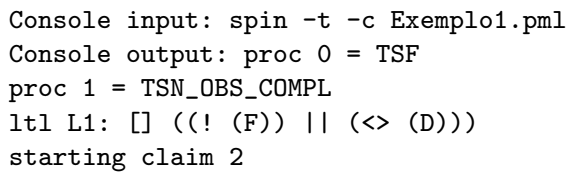

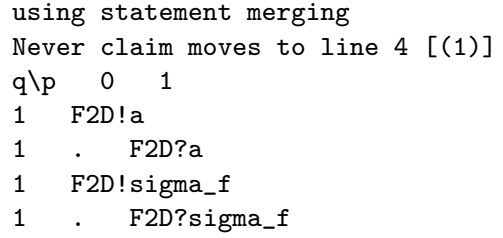

\section{CONCLUSIONS AND FUTURE WORKS}

In this paper we presented a model checking approach to deal with diagnosability of DES, our approach is quite different from the ones found in the literature where they direct test if a given DES is diagnosable with respect to a set of failures and observations. In our approach we propose an indirect test, first we construct a Transition System that indicates when an observed run goes out of the normal behavior of the system and then perform model checking in the model that represents the interaction between it with the faulty runs of the system, using LTL to express that whenever a fault occur in the system it should be always diagnosed in the future. One advantage of this approach is that by our examination and tests, we conjecture that it is quite general, and could be applied to check diagnosability of timed and hybrid systems. Another advantage of our approach is that it can be easily applied in state of the art model checkers such as SPIN with minors adaptations. SPIN is a quite powerful model checking tool and have been applied in several interesting study cases. In future works we intend to perform a benchmarking between our proposed approach and others found in the literature in order to better evaluate the potential of our. We also expect to extend this approach to deal with timed and hybrid system.

\section{REFERENCES}

Baier, C. and Katoen, J.P. (2008). Principles of Model Checking. MIT Press.

Cassandras, C.G. and Lafortune, S. (2008). Introduction to Discrete Event Systems. Springer, Massachussets, 2 edition.

Cassez, F. (2012). The complexity of codiagnosability for discrete event and timed systems. IEEE TRANSACTIONS ON AUTOMATIC CONTROL, 57(7), 1752-1764.

Clarke, E.M., Henzinger, T.A., Veith, H., and Bloem, R. (2018). Handbook of Model Checking, volume 1. Springer International Publishing.

Jiang, S. and Kumar, R. (2004). Failure diagnosis of discrete-event systems with linear-time temporal logic specifications. IEEE Transactions on Automatic Control, 49(6), 934-945. doi:10.1109/TAC.2004.829616.

Jiang, S. and Kumar, R. (2006). Diagnosis of repeated failures for discrete event systems with linear-time temporal-logic specifications. IEEE Transactions on Automatic Control, 3(1), 47-58.

Sampath, M., Sengupta, R., Lafortune, S., Sinnamohideen, K., and Teneketzis, D. (1995). Diagnosability of discreteevent systems. IEEE Transactions on Automatic Control, 40(9), 1555-1575. doi:10.1109/9.412626.

Vardi, M.Y. and Wolper, P. (1986). An automatatheoretic approach to automatic program verification (preliminary report). 1st Annual Symposium on Logic in Computer Science (LICS), 332-334. 